\title{
HIV infection and sexual behaviour in primary and secondary infertile relationships: a case-control study in Kigali, Rwanda
}

\author{
Nathalie Dhont, ${ }^{1,2}$ Claude Muvunyi, ${ }^{3,4}$ Stanley Luchters, ${ }^{1}$ Joseph Vyankandondera, ${ }^{2,5}$ \\ Ludwig De Naeyer, ${ }^{6}$ Marleen Temmerman, ${ }^{1}$ Janneke van de Wijgert ${ }^{7,8}$
}

${ }^{1}$ International Centre for Reproductive Health, Ghent University, Ghent, Belgium ${ }^{2}$ Projet Ubuzima, Kigali, Rwanda ${ }^{3}$ Microbiology Department, Ghent University, Ghent, Belgium

${ }^{4}$ National University of Rwanda, Microbiology Department, Butare, Rwanda

${ }^{5}$ Belgian Technical Cooperation, Rwanda

${ }^{6}$ Management Sciences for Health, Rwanda

${ }^{7}$ Academic Medical Centre of the University of Amsterdam, Department of Internal

Medicine, The Netherlands

${ }^{8}$ Institute for Global Health and Development, Amsterdam, The Netherlands

\section{Correspondence to}

Dr N Dhont, c/o Projet Ubuzima, Rue Akagera n 715, Kiyovu, Kigali, Rwanda;

dhontnathalie@gmail.com

Accepted 3 August 2010

Published Online First

18 September 2010

\begin{abstract}
Objective To compare the prevalence of sexually transmitted infections (STIs) (including HIV) and of highrisk sexual behaviour in the following three groups: primary infertile relationships, secondary infertile relationships and fertile relationships. Primary infertility is here defined as never having conceived before, secondary infertility as infertility subsequent to having conceived at least once. Design Unmatched case-control study.

Methods Sexually active infertile women aged 21-45 years presenting at an infertility clinic of the Kigali Teaching Hospital, Rwanda and their male partners were invited to participate. Fertile controls who had recently delivered were recruited from the community. In a face-to-face interview, participants were asked about sociodemographic characteristics and their sexual behaviours, and tested for HIV and STIs.
\end{abstract}

Results Between November 2007 and May 2009, 312 women and 254 partners in infertile relationships and 312 women and 189 partners in fertile relationships were enrolled. Involvement in a secondary infertile relationship was associated with HIV infection after adjusting for sociodemographic covariates for women (adjusted OR $(A O R)=4.03,95 \% \mathrm{Cl} 2.4$ to 6.7$)$ and for men $(A O R=3.3$, $95 \% \mathrm{Cl} 1.8$ to 6.4$)$. Involvement in a primary infertile relationship, however, was not. Secondary infertile women were more likely to have engaged in risky sexual behaviour during their lifetime compared with primary infertile and fertile women. Men in primary and secondary infertile relationships more often reported multiple partners in the past year $(A O R=5.4,95 \% \mathrm{Cl} 2.2$ to 12.7 ; $\mathrm{AOR}=7.1,95 \% \mathrm{Cl} 3.2$ to 15.8 , respectively).

Conclusions Increased HIV prevalence and risky sexual behaviour among infertile couples is driven by secondary infertility. Infertile couples, and especially those with secondary infertility, should be targeted for HIV prevention programmes and their fertility problems should be addressed.

\section{INTRODUCTION}

Infertility and HIV infection are affecting a large number of couples world wide with sub-Saharan Africa bearing the largest burden of these two reproductive disorders. It is estimated that in this region $2.5 \%$ of adults are living with HIV. $^{1}$ Primary infertility (never having conceived) is affecting $3-6 \%$ of couples, whereas the prevalence of secondary infertility (infertility after having conceived at least once before) is much higher, especially in certain regions, affecting $5-25 \%$ of couples. ${ }^{2}$
A number of studies have shown an association between infertility and HIV infection, focusing mainly on reduced fecundity among HIV-positive women compared with HIV-negative women HIV-positive women (also those with asymptomatic infection) have lower conception rates and higher rates of pregnancy loss. The lower conception rate is thought to be due to greater susceptibility to pelvic inflammatory disease with resultant tubal factor infertility and to weight loss related anovulation, amenorrhoea, male hypogonadism and impaired spermatogenesis. ${ }^{3-9}$ However, in one prospective study examining the association between HIV infection and infertility, it was found that in half of the patients the infertility had existed before HIV infection, suggesting that infertility is a risk factor for HIV acquisition. ${ }^{10}$ Previous studies in sub-Saharan Africa have shown that couples with fertility problems are likely to face extramarital relationships, polygamous unions and divorce, all of which are known risk factors for HIV infection. ${ }^{11-14}$ Few studies have examined the HIV prevalence and current sexual behaviours in infertile patients, particularly among couples. In Tanzania, HIV infection was threefold higher among infertile women than among fertile controls, and perceived partner infidelity was higher among infertile women. ${ }^{15}$ Similarly, in Nigeria, HIV infection among women undergoing laparoscopy evaluation for infertility was fivefold higher than HIV prevalence among pregnant women. ${ }^{16}$

Most HIV transmission in sub-Saharan Africa is thought to be through heterosexual transmission among HIV discordant couples in stable relationships. ${ }^{17} 18$ Couples in infertile relationships may constitute an important vulnerable group for HIV infection in African society and may fuel the HIV epidemic. Little is known about the association between infertility, risky sexual behaviour and sexually transmitted infections (STIs), including HIV. We therefore examined whether HIV/STI infection and risky sexual behaviour were more common among men and women in primary and secondary infertile relationships than among men and women in fertile relationships in an urban population in Kigali, Rwanda.

\section{MATERIAL AND METHODS \\ Study population and setting}

Between November 2007 and May 2009, an infertility research clinic was opened at the Kigali Teaching Hospital in Rwanda (the largest public hospital in Rwanda), and infertile women were 
recruited mainly through word of mouth. Recruitment was terminated when 312 infertile women were enrolled, as per protocol. To be eligible for participation, women needed to be between 21 and 45 years of age, residing in Kigali, willing to undergo HIV testing and to have had sexual intercourse at least once in the past 2 weeks. Refusal to participate in the study did not influence access to further services. Infertility was defined as failure to conceive after regular unprotected intercourse for $\geq 1$ year and included primary and secondary infertility. Male partners of primary and secondary infertile women are referred to as men in primary and secondary infertile relationships, respectively. In the analyses presented in this paper, infertility was considered a problem for couples and no distinction was made between female or male causes of infertility. Fertile controls were defined as women who delivered between 6 and 18 months ago. Other eligibility criteria were the same as for the infertile women. Controls were recruited from the community, as no appropriate control group could be selected at the hospital. A list of all residential neighbourhoods (known as umudugudu) from where the infertile couples originated was compiled (120 umudugudus in total). Fourteen neighbourhoods were randomly selected from this list by blindly hand picking numbers from a bowl. Community mobilisers in these neighbourhoods visited all families to identify potential eligible candidates.

After inclusion, all cases and controls invited their male partners to participate on a separate occasion.

All participating women were offered free cervical cancer screening and treatment when clinically indicated. The study was approved by the National Ethics Committee of Rwanda, the ethics committee of the University Hospital Ghent and the National AIDS Control Program (CNLS) in Rwanda.

\section{Procedures}

The purpose of the study was explained to potential participants in their local language (Kinyarwanda) and eligibility criteria were checked. All participants provided written informed consent before the study procedures took place. Women were interviewed by a female nurse and male partners by a male medical officer about sociodemographic characteristics, sexual behaviour and obstetric history using structured questionnaires. Some of the female participants ( 30 women) were asked again about extramarital partners in a follow-up interview when positive STI results were discussed. Female participants received a clinical gynaecological examination by the female nurse, and male participants a genital examination by the male medical officer; all clinical procedures were supervised by a gynaecologist. During the speculum examination, clinical signs of STIs were documented, vaginal $\mathrm{pH}$ was measured, specimens for STI diagnostic tests were collected, and visual inspection with acetic acid was performed to identify cervical precancerous lesions. Saline wet mounts were examined at the study clinic for the presence of motile Trichomonas vaginalis. A second vaginal swab was placed in $3 \mathrm{ml}$ UTM-RT transport medium (Copan Diagnostics, California, USA) and immediately frozen at $-80^{\circ} \mathrm{C}$. This swab was shipped, frozen on dry ice, to Ghent University Hospital for multiplex PCR for $C$ trachomatis and $N$ gonorrhoea (Abbott Laboratories, Illinois, USA).

After the speculum examination and male genital examination, blood samples were collected for on-site serial rapid HIV testing according to a national algorithm (Determine Rapid HIV-1/2 Test (Abbott Laboratories), followed by Uni-Gold Rapid Test (Trinity Biotech, Ireland) if determined to be positive, and followed by Capillus HIV-1/HIV-2 Rapid Test (Trinity Biotech Plc, Ireland), if a tiebreaker was needed, and an on-site
Treponema-specific rapid syphilis test (SD Bioline Syphilis 3.0, Standard Diagnostics, Korea). If the rapid syphilis test was positive, rapid plasma reagin (RPR) titration (Human, Germany) was carried out in the National Reference Laboratory in Kigali. A positive Treponema-specific rapid test with a positive RPR was interpreted as active or recently treated syphilis, while a positive Treponema-specific rapid test and negative RPR was considered evidence of old previously treated syphilis. The serum sample was immediately processed in four aliquots and stored at $-80^{\circ} \mathrm{C}$ until analysis. One aliquot was analysed for HSV-2 antibodies (Herpeselect 2 ELISA IgG, Focus Diagnostics, California, USA) in the National Reference Laboratory. Participants were advised to return for test results and, if required, received treatment according to local guidelines. All participants were offered HIV counselling and same-day testing; those testing positive were enrolled in an HIV care and treatment clinic of their choice.

Infertile couples were investigated with a tubal patency test (hysterosalpingography or laparoscopy) and a semen analysis.

\section{Variables and study measures}

Sexual behaviour was assessed with the following indicators: ever separated, lifetime sexual partners, age at first intercourse, ever engaged in transactional sex (women only), ever use of condoms, multiple partners in past year (concurrent partnerships and/or more than one partner in the past year), perception of partner's fidelity and condom use at last sexual intercourse (men only). STIs included self-reported STIs (any genital symptoms within the past year), clinically diagnosed STIs (genital ulcers/warts/blisters, urethral discharge, cervicitis and pelvic inflammatory disease) and STIs diagnosed using laboratory tests ( $T$ vaginalis, $N$ gonorrhoeae, $C$ trachomatis and $T$ pallidum, HIV and herpes simplex virus 2 (HSV-2) serology). STI laboratory tests among men were limited to $T$ pallidum, HIV and HSV-2 serology.

\section{Statistical analysis}

Data collected during interviews and laboratory investigations were single entered, verified and cleaned using MS Access 2000 (Microsoft). Intercooled Stata 9.2 (Stata Corporation) was used for statistical analysis. Sociodemographic, sexual behaviour and reproductive health characteristics of infertile and fertile women and their partners were compared using $\chi^{2}$ tests or Mann-Whitney $U$ tests. Crude and adjusted ORs were calculated for men and women separately to compare HIV prevalence, sexual behaviour and STI prevalence. Potential confounding variables among the sociodemographic characteristics were selected a priori and retained in multivariable models when they affected the association (OR) between the primary predictor of interest and the outcome by $\geq 10 \%$.

As a result, all primary predictors in women were controlled for age, occupation, income and marital status, in men predictors were controlled for age, income and education.

\section{RESULTS}

\section{Baseline characteristics}

At total of 312 female cases and 312 female controls were recruited. Three hundred and thirty-nine infertile women presented at the clinic, of whom 312 eligible women were subsequently enrolled. Community mobilisers identified and invited 407 fertile women after visiting all families in selected neighbourhoods. Of these, 55 women were not interested in participating owing to lack of time, fear of the gynaecological examinations, or an unknown reason. The remaining 352 women came to the clinic (response rate of $86 \%$ ). Of these, 
40 women were ineligible and 312 eligible fertile women subsequently enrolled. Educational level and occupation did not differ between responders and non-responders.

Male partners of $81 \%$ of the infertile women (254 partners) and $61 \%$ of the fertile women (189 partners) agreed to participate. Of women in infertile relationships, 135 (43\%) had primary infertility and 177 (57\%) secondary infertility. Primary infertile women were more likely to be married then fertile women (table 1). Both primary and secondary infertile women were more likely to be older, and to have an occupation and an income, and less likely to have had an HIV test in their lifetime than fertile women. All unmarried participants in the three groups reported having a steady sexual partner, and in most cases $(90 \%)$, this partner spent the night with them most of the time. Men in both primary and secondary infertile relationships were older; more educated and were less likely to have had an HIV test in their lifetime than men in fertile relationships.

\section{Sexual risk behaviour and STIs in women}

Primary infertile women were more likely to ever have separated from a steady sexual partner $(A O R=2.03,95 \%$ CI 1.07 to 3.84 ), to have had more than three lifetime sexual partners $(\mathrm{AOR}=3.01,95 \% \mathrm{CI} 1.75$ to 5.16$)$ and to never have used condoms than fertile women ( $\mathrm{AOR}=3.53,95 \% \mathrm{CI} 2.21$ to 5.62 ) (table 2). Secondary infertile women had higher levels of risky sexual behaviour than fertile women, including ever having been separated ( $\mathrm{AOR}=3.27,95 \% \mathrm{CI} 1.94$ to 5.52), lower age at first intercourse ( $\mathrm{AOR}=1.68,95 \% \mathrm{CI} 1.08$ to 2.60$)$, a higher number of lifetime sexual partners ( $\mathrm{AOR}=6.74,95 \% \mathrm{CI} 4.12$ to 11.03 ) and ever having engaged in transactional sex $(\mathrm{AOR}=2.98,95 \%$ CI 1.39 to 6.41). Separations were mostly a result of childlessness for primary infertile women but not for secondary infertile women. Multiple partners in the past year were infrequently reported by all three groups of women, but statistically significantly more often by secondary infertile women $(\mathrm{AOR}=5.66$, $95 \%$ CI 2.10 to 15.27 ), and slightly more often by primary infertile women ( $\mathrm{AOR}=2.82$, 95\% CI 0.84 to 9.46) compared with fertile women. Primary and secondary infertile women were more likely to believe that their partners had another partner $(\mathrm{AOR}=4.13,95 \% \mathrm{CI} 2.03$ to $8.41 ; \mathrm{AOR}=4.85,95 \% \mathrm{CI}$ 2.55 to 9.20 , respectively) than fertile women. Thirty women with a positive test result for $N$ gonorrhoea and/or $C$ trachomatis were asked again about their number of sexual partners in the past year and their responses did not differ from those given during the initial interview.

No difference in the prevalence of STIs was found between primary infertile women and fertile women. However, secondary infertile women were more likely to have laboratory evidence of an old previously treated syphilis infection ( $\mathrm{AOR}=2.32$, 95\% CI 1.00 to 5.40 ), positive HSV-2 serology (AOR $=2.59,95 \%$ CI 1.65 to 4.05$)$ and to have reported genital symptoms in the past year ( $\mathrm{AOR}=3.98,95 \% \mathrm{CI} 0.1 .71$ to 9.29 ) than fertile women. The prevalence of any active STI (including clinical STIs, active syphilis, gonorrhoea, chlamydia and trichomoniasis) did not differ between the three groups.

HIV prevalence was $16 \%$ among infertile women, $18 \%$ among primary infertile women and $43 \%$ among secondary infertile women. Having secondary infertility but not primary infertility was associated with HIV infection for women (AOR=4.03, 95\% CI 2.44 to $6.65 ; \mathrm{AOR}=1.63,95 \%$ CI 0.90 to 2.96 , respectively).

Sexual behaviours and STI prevalence differed significantly between primary and secondary infertile women. Except for the reporting of partner's infidelity, all indicators of risky sexual behaviours were reported more frequently, and the prevalence of
STIs, except for any active STI, was higher among secondary infertile women than among primary infertile women.

Tubal factor infertility was diagnosed in $61 \%$ of primary infertile women and in $73 \%$ of secondary infertile women $(\mathrm{p}=0.03)$ (data not shown).

\section{Sexual risk behaviour and STIs in men}

Men in primary infertile relationships were more likely to never have used condoms ( $\mathrm{AOR}=2.30,95 \% \mathrm{CI} 1.37$ to 3.85 ) and men in secondary infertile relationships were more likely to have separated at least once $(\mathrm{AOR}=2.03,95 \% \mathrm{CI} 1.07$ to 3.84$)$ compared with fertile men (table 3 ). Men in primary and secondary infertile relationships were more likely to report multiple sexual partners in the past year $(A O R=5.34,95 \%$ CI 2.24 to $12.72 ; \mathrm{AOR}=7.11,95 \%$ CI 3.20 to 15.78 , respectively) and lack of condom use at last sexual intercourse $(\mathrm{AOR}=8.51$, 95\% CI 1.91 to $37.92 ; \mathrm{AOR}=3.03,95 \%$ CI 1.16 to 7.87 , respectively) than men in fertile relationships. Of 25 men in an infertile relationship who knew their HIV-positive status before the study, only two reported condom use at last sexual intercourse compared with 11 out of 13 men in a fertile relationship. Only three men (two in a fertile and one in a primary infertile relationship) believed that their female partners had another sexual partner. Prevalence of STIs did not differ between men in primary infertile relationships and men in fertile relationships. Men in a secondary infertile relationship were more likely to have a positive HSV-2 serology than fertile men ( $\mathrm{AOR}=2.57$, $95 \%$ CI 1.58 to 4.16 ). The reporting of genital symptoms and the presence of any active STI was slightly higher in men in secondary infertile relationships but did not reach statistical significance.

In men, HIV infection was statistically significantly associated with being in a secondary infertile relationship ( $\mathrm{AOR}=3.28,95 \%$ CI 1.78 to 6.41), and non-significantly associated with being in a primary infertile relationships $(A O R=1.86,95 \%$ CI 0.90 to 3.85 ).

Apart from never use of condoms, which was reported more frequently by men in primary infertile relationships, levels of sexual activity in past and present did not differ between men in primary and secondary infertile relationships.

In $64 \%$ of men in primary and $68 \%$ of men in secondary infertile relationships abnormal semen parameters or sexual dysfunction were found.

\section{HIV diagnosis on couple level}

Couples in secondary infertile relationships were less likely to be seroconcordant negative, more likely to be seroconcordant positive and more likely to be serodiscordant with a positive female partner (table 4). Little difference was seen in the result of the HIV test between couples in primary infertile relationships and those in fertile relationships, apart from a higher tendency among the former to be seroconcordant positive.

\section{DISCUSSION}

Our study showed that increased HIV prevalence among infertile couples is driven by secondary infertile relationships. Secondary infertile women exhibited a higher-risk sexual behaviour profile in their lifetime than primary infertile and fertile women. Furthermore, men in both primary and secondary infertile relationships were more likely to report multiple partners in the past year than men in fertile relationships.

A higher HIV prevalence among infertile couples has been reported by three previous studies, but these studies did not include data on male partners. A study in Tanzania found 
Table 1 Characteristics of enrolled women and their male partners in fertile and infertile relationships and by infertility type in Kigali, Rwanda

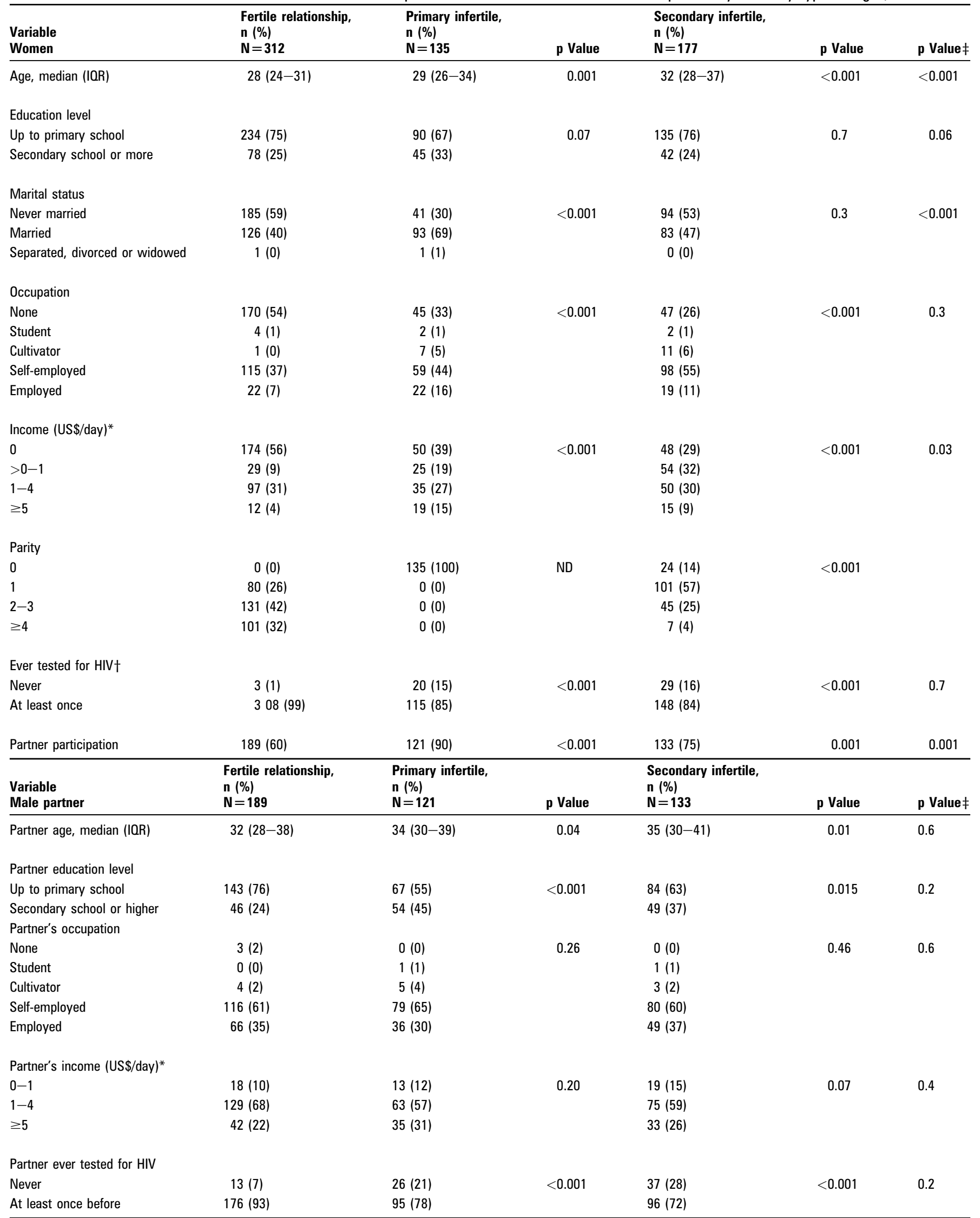

\footnotetext{
* Six and 10 missing values for primary and secondary infertile women and 10 and six for men in primary and secondary infertile relationships.

tOne missing value for fertile relationship.

$\ddagger$ Derived from comparison between primary and secondary infertile relationships.
} 
Table 2 Sexual behaviour and sexually transmitted infections (STIs) among women in fertile, primary infertile and secondary infertile relationships

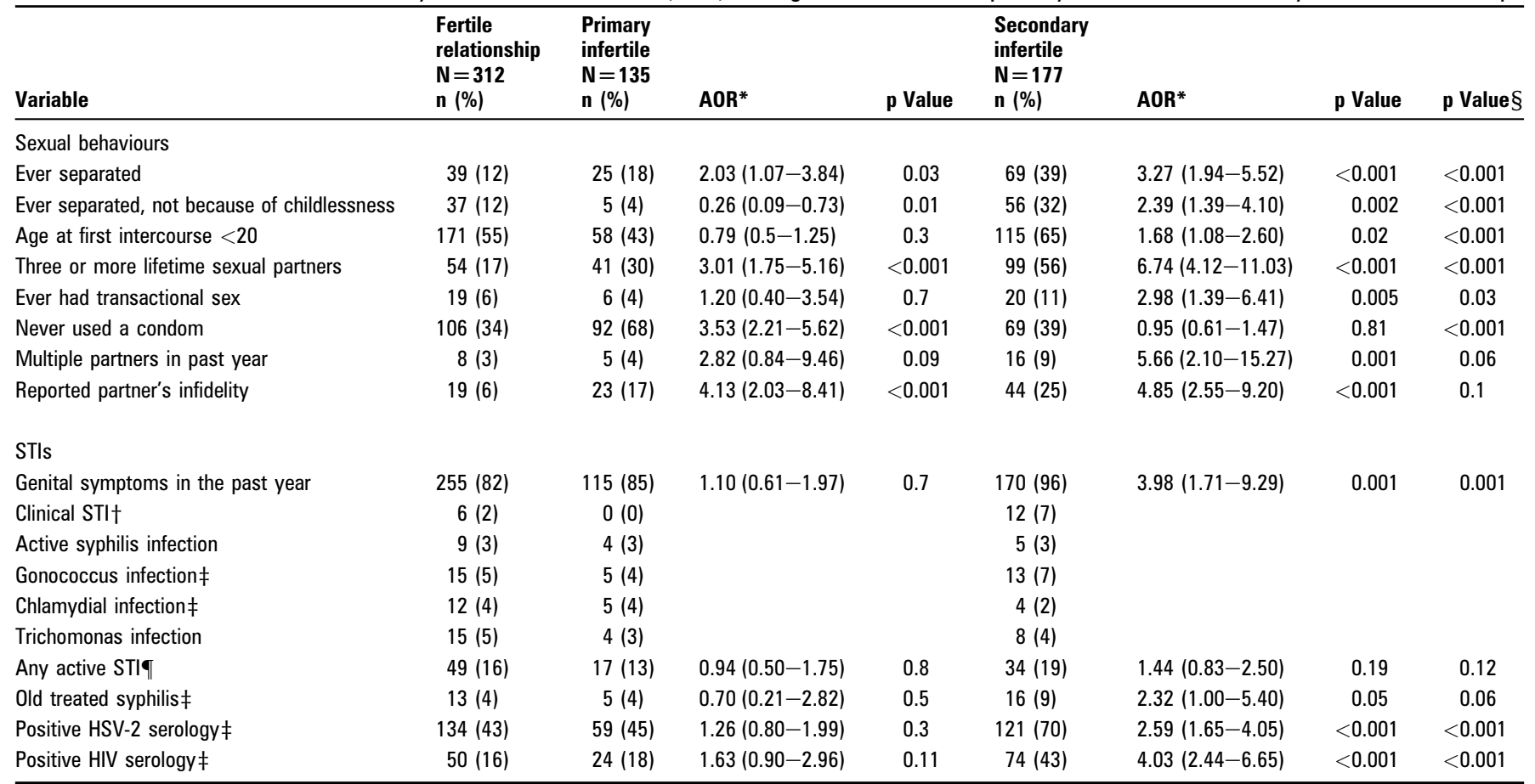

*Adjusted for female age, occupation, marital status and income in logistic regression model, no other variables are included

†Includes cervicitis, pelvic inflammatory disease and genital ulcers/warts/blister.

$¥$ Syphilis: two and three cases missing for primary and secondary infertility respectively; HSV-2: three cases missing for fertile relationships, four cases for primary and secondary infertility; HIV: three cases missing for primary and secondary infertility; Chlamydia: 11 cases missing for fertile relationships; Gonorrhoea: 11 cases missing for fertile relationships and one for secondary infertility.

§Last column $\mathrm{p}$ value is derived from comparison of primary and secondary infertile women.

TIncludes clinical STIs, active syphilis, gonococcus, chlamydial and trichomonas infection.

AOR, adjusted odds ratio; HSV-2, herpes simplex virus 2.

a threefold higher HIV prevalence among infertile women, a higher exposure to STIs in the past, but no difference for current STIs compared with pregnant women. Two studies in Nigeria reported a higher HIV prevalence among infertile women undergoing diagnostic evaluation and among women with tubal infertility compared with the general population. ${ }^{3} 16$ The HIV prevalence of $16 \%$ among fertile women in our study agrees with the reported HIV prevalence of $15.4 \%$ among

Table 3 Sexual behaviour and sexually transmitted infections (STIs) among men in fertile, primary infertile and secondary infertile relationships

\begin{tabular}{|c|c|c|c|c|c|c|c|c|}
\hline Variable & $\begin{array}{l}\text { Fertile } \\
\text { relationship } \\
\mathrm{N}=189 \\
\mathrm{n}(\%)\end{array}$ & $\begin{array}{l}\text { Primary } \\
\text { infertile } \\
N=121 \\
n(\%)\end{array}$ & $A O R^{*}$ & p Value & $\begin{array}{l}\text { Secondary } \\
\text { infertile } \\
N=133 \\
n(\%)\end{array}$ & $A O R^{*}$ & p Value & p Value $\S$ \\
\hline \multicolumn{9}{|l|}{ Sexual behaviours } \\
\hline Ever separated & $22(12)$ & $26(21)$ & $1.66(0.83-3.31)$ & 0.15 & $32(24)$ & $2.03(1.07-3.84)$ & 0.03 & 0.6 \\
\hline Ever separated (not because of childlessness) & $21(11)$ & $26(21)$ & $1.80(0.89-3.61)$ & 0.10 & $29(22)$ & $1.82(0.94-3.52)$ & 0.07 & 0.9 \\
\hline Age at first intercourse $<20$ & 79 (42) & $53(44)$ & $1.00(0.96-1.03)$ & 0.9 & 65 (49) & $0.97(0.93-1.00)$ & 0.06 & 0.4 \\
\hline Four or more lifetime sexual partners & $86(45)$ & 57 (47) & $0.95(0.81-1.12)$ & 0.5 & $76(57)$ & $1.12(0.96-1.31)$ & 0.14 & 0.1 \\
\hline Never used a condom & $47(25)$ & $49(41)$ & $2.30(1.37-3.85)$ & 0.002 & $38(29)$ & $1.18(0.70-2.00)$ & 0.5 & 0.05 \\
\hline No condom use at last sexual intercourse & $167(88)$ & $119(98)$ & $8.51(1.91-37.92)$ & 0.005 & $127(95)$ & $3.03(1.16-7.87)$ & 0.02 & 0.2 \\
\hline Multiple partners in past year & $9(5)$ & $19(16)$ & $5.34(2.24-12.72)$ & $<0.001$ & $32(24)$ & $7.11(3.20-15.78)$ & $<0.001$ & 0.1 \\
\hline Reported partner's infidelity & $2(1)$ & $1(1)$ & & & $0(0)$ & & & \\
\hline \multicolumn{9}{|l|}{ STIs } \\
\hline Genital symptoms in the past year & $13(7)$ & $13(11)$ & $1.51(0.63-3.59)$ & 0.3 & $16(12)$ & $1.89(0.85-4.21)$ & 0.12 & 0.7 \\
\hline Clinical STI† & $7(4)$ & $2(2)$ & & & $9(7)$ & & & \\
\hline Active syphilis infection $\neq$ & $2(1)$ & $3(2)$ & & & $3(2)$ & & & \\
\hline Any active STIף & $9(5)$ & $5(4)$ & $0.70(0.20-2.44)$ & 0.6 & $12(9)$ & $2.30(0.89-5.92)$ & 0.08 & 0.1 \\
\hline Old treated syphilis $\ddagger$ & $10(5)$ & $7(6)$ & $1.44(0.52-3.99)$ & 0.5 & $3(2)$ & $0.48(0.13-1.84)$ & 0.3 & 0.1 \\
\hline Positive HSV-2 serology $\ddagger$ & $71(38)$ & $50(42)$ & $1.27(0.77-2.09)$ & 0.3 & $79(60)$ & $2.57(1.58-4.16)$ & $<0.001$ & 0.005 \\
\hline Positive HIV serology $\ddagger$ & $18(9)$ & $19(16)$ & $1.86(0.90-3.85)$ & 0.09 & $36(27)$ & $3.28(1.78-6.41)$ & $<0.001$ & 0.03 \\
\hline
\end{tabular}

${ }^{*}$ Adjusted for male age, education and income.

†Clinical STIs include urethral discharge and genital ulcer; one case missing for fertile men.

¥Syphilis: one case missing for secondary infertility; HSV-2: two and one case missing for primary and secondary infertility, respectively; HIV: one case missing for secondary infertility.

§Last column $\mathrm{p}$ value derived from comparison of men in primary and secondary infertile relationships.

qIncludes active syphilis infection and clinical STIs.

AOR, adjusted odds ratio; HSV-2, herpes simplex virus 2. 
Table 4 HIV diagnosis on couple level ${ }^{*}$ according to fertility status

\begin{tabular}{lcccccc}
\hline & $\begin{array}{l}\text { Fertile } \\
\mathbf{N = 1 8 9} \\
\mathbf{n}(\%)\end{array}$ & $\begin{array}{l}\text { Primary } \\
\text { infertile } \\
\mathbf{N = 1 1 9} \\
\mathbf{n}(\%)\end{array}$ & p Value & $\begin{array}{l}\text { Secondary } \\
\text { infertile } \\
\mathbf{N = 1 3 2}\end{array}$ & \\
$\mathbf{n}(\%)$ & p Value \\
\hline Bariable & $155(82)$ & $94(79)$ & 0.5 & $73(55)$ & $<0.001$ \\
Both seronegative & $12(6)$ & $14(12)$ & 0.1 & $27(20)$ & $<0.001$ \\
Serodiscordant (female +$)$ & $16(8)$ & $6(5)$ & 0.3 & $23(17)$ & 0.02 \\
Serodiscordant (male +$)$ & $6(3)$ & $5(4)$ & 0.6 & $9(7)$ & 0.1 \\
\hline
\end{tabular}

*Only the couples in whom HIV testing was completed are included.

women attending antenatal clinics in Kigali in 2003. ${ }^{19}$ The higher HIV prevalence among couples in secondary infertile relationships contrasts with previous reports showing the same or a higher HIV prevalence in primary infertile women compared with secondary infertile women. ${ }^{15} 20$ Our data suggest that secondary infertile women represent a distinct group of infertile women characterised by a risky sexual behaviour profile, often starting in adolescence, which has predisposed them to a first pregnancy (the majority of them had had only one pregnancy), HIV infection, other STIs and subsequent infertility. It is of interest to note that $55 \%$ of the secondary infertile women had their first pregnancy before the age of 21 (marriage before the age of 21 is illegal in Rwanda) and $28 \%$ had experienced at least one unwanted pregnancy. These proportions were significantly lower in the fertile women in our study (N Dhont, unpublished data). The profile of secondary infertile women shows that the epidemics of HIV, STIs, unintended pregnancies and (secondary) infertility are closely linked. Primary infertile women do not report a higher-risk sexual behaviour than fertile women, apart from never use of condoms, which is likely to be a result of their infertility, and a higher number of lifetime partners, which is in part related to unstable relationships caused by infertility. It can be hypothesised that it is rather the risky sexual behaviour of their current and/or past sexual partner that has predisposed them to infertility.

We did not find a higher prevalence of any active STI among infertile couples than among fertile controls despite the higher level of reporting recent high-risk sexual behaviour. This might in part be due to the more vigorous treatment-seeking behaviour among women in infertile relationships when facing genital symptoms. Of the women in infertile relationships, $74 \%$ had sought treatment for the last STI episode versus $50 \%$ for women in fertile relationships. This phenomenon has been described elsewhere. ${ }^{15}$ Another study in Nigeria did find a higher prevalence of cervical $N$ gonorrhoea in secondary infertile women than in primary infertile and fertile women. ${ }^{21}$ Unfortunately, we could not implement extensive STI testing in male participants.

It should be noted that selection bias may have influenced the results of our study. The infertile study population is a selection of couples who are willing to undergo infertility investigations; they do not necessarily represent all infertile couples in Kigali. Married infertile couples may have been more motivated to participate in this study than non-married infertile couples because marriage often represents a higher expectation to produce children than non-legalised cohabitation. Married women are less likely to report extramarital sex, and overrepresentation of married women in the infertile group may therefore have resulted in an underestimation of the HIV-risk behaviours in this group. The different participation rates of male partners in all three groups may also have induced a selection bias. It is possible that disproportionately more faithful fertile men participated in the study, which might have led to overestimation of the differences in HIV-risk behaviour between men in fertile and infertile relationships. However, male infidelity was also reported consistently more frequently by infertile women than by fertile women.

Conclusions about the temporality or causality of the relationships between infertility, sexual behaviour and HIV infection cannot be drawn owing to the case-control design of the study and the lack of data on timing of HIV infections (such as $\mathrm{CD} 4+$ counts). However, our own observations and other sources of information are in favour of a causal role of infertility in sexual behaviour and HIV infections. Several social science studies have shown that infertility leads to the practice of 'looking elsewhere for offspring', especially among men and to a lesser extent women, and to unstable sexual relationships. ${ }^{11} 1222$ These behaviours in turn predispose women and men to HIV infection and STIs. The risky sexual behaviour which put women, and to a lesser extent men, at risk for infertility is perpetuated or might be reinforced after they have become infertile, closing a vicious circle of risky sexual behaviour, HIV infection and infertility.

Furthermore, in our study, high-risk male sexual behaviour in the past was only weakly associated with infertility, whereas more recent high-risk behaviour was more strongly associated with infertility, suggesting that their current sexual behaviour could be a consequence of infertility.

Finally, we found an association, although not statistically significant, between HIV infection and longer infertility duration ( $>5$ years) after adjusting for age.

Our study has several implications. First, HIV prevention programmes should target infertile couples, especially secondary infertile, as a high-risk group. Voluntary HIV counselling and testing of infertile couples may identify new HIV infections and increase opportunities for HIV care and prevention. Young people should be taught that unsafe sex increases the risk of acquiring HIV and unintended pregnancies, and also can lead to infertility. Second, provision of fertility services (including safe services for women who are already HIV-infected) could reduce high-risk behaviours and reduce HIV transmission. Some fertility services are readily available in developing countries but are underused, and others are currently not available. To increase access, fertility services could be integrated in family planning or women's health clinics. Finally, more research is needed to determine the prevalence of primary and secondary infertility in different parts of sub-Saharan Africa, and the temporal relationships between infertility, sexual behaviour and HIV infection.

\section{Key messages}

- In men and women, HIV prevalence rates are significantly higher for secondary infertile relationships than for either primary infertile relationships or fertile relationships.

- In women, secondary infertility is associated with a greater lifetime probability of high-risk sexual behaviour than primary infertility and fertility.

- In men, both primary and secondary infertile relationships are associated with a higher rate of reported multiple partners than fertile relationships.

- Infertility in sub-Saharan Africa should be addressed because it generates enormous suffering and also is closely linked to the HIV epidemic and hampers HIV prevention efforts. 
Acknowledgements We thank Rosette Busasa, Ammiel Gasarabwe and the Community Mobilisers for their dedication during data collection, Dr Gilles Ndabisaya for programming the database, and other members of the Projet Ubuzima team and the EDCTP microbicides project team for their contributions and support.

Funding This study was funded by a PhD grant from the Flemish Interuniversity Council (VLIR-UOS). The project also received funding from the European and Developing Countries Clinical Trials Partnership (EDCTP) through a project entitled: 'Preparing for phase III vaginal microbicide trials in Rwanda and Kenya: preparedness studies, capacity building and strengthening of medical referral systems.' However, EDCTP cannot accept any responsibility for information or views expressed herein.

\section{Competing interests None.}

Patient consent Obtained.

Ethics approval This study was conducted with the approval of the Rwanda National Ethics Committee and ethics committee of the University Hospital Ghent.

Contributors The study was conceived and designed by ND, MT and JvdW, JV. ND, $\mathrm{CM}$ and JV contributed to specimen and data collection. ND analysed the study data and drafted the article. SL, LDN and JvdW assisted with data analysis and interpretation of data. CM, LDN, SL and JV revised the article critically. MT and JvdW provided overall supervision and critical revision of the article.

Provenance and peer review Not commissioned; externally peer reviewed.

\section{REFERENCES}

1. Hunter DJ. AIDS in sub-Saharan Africa: the epidemiology of heterosexual transmission and the prospects for prevention. Epidemiology 1993;4:63-72.

2. Larsen U. Primary and secondary infertility in sub-Saharan Africa. Int J Epidemiol 2000;29:285-91.

3. Adesiyun AG, Ameh CA, Eka A. Hysterosalpingographic tubal abnormalities and HIV infection among black women with tubal infertility in sub-Saharan Africa. Gynecol Obstet Invest 2008;66:119-22.

4. Cohen CR, Sinei S, Reilly $M$, et al. Effect of human immunodeficiency virus type 1 infection upon acute salpingitis: a laparoscopic study. J Infect Dis 1998;178:1352-8.

5. Gray RH, Wawer MJ, Serwadda D, et al. Population-based study of fertility in women with HIV-1 infection in Uganda. Lancet 1998;351:98-103.
6. Kamenga MC, De Cock KM, St Louis ME, et al. The impact of human immunodeficiency virus infection on pelvic inflammatory disease: a case-control study in Abidjan, Ivory Coast. Am J Obstet Gynecol 1995;172:919-25.

7. Moodley P, Wilkinson D, Connolly $\mathrm{C}$, et al. Trichomonas vaginalis is associated with pelvic inflammatory disease in women infected with human immunodeficiency virus. Clin Infect Dis 2002:34:519-22.

8. Temmerman M, Lopita Ml, Sanghvi HC, et al. The role of maternal syphilis, gonorrhoea and HIV-1 infections in spontaneous abortion. Int J STD AIDS 1992;3:418-22.

9. Nicopoullos JD, Almeida PA, Ramsay JW, et al. The effect of human immunodeficiency virus on sperm parameters and the outcome of intrauterine insemination following sperm washing. Hum Reprod 2004;19:2289-97.

10. Ross A, Morgan D, Lubega $R$, et al. Reduced fertility associated with HIV: the contribution of pre-existing subfertility. AIDS 1999;13:2133-41.

11. Dyer SJ, Abrahams N, Hoffman M, et al. 'Men leave me as I cannot have children': women's experiences with involuntary childlessness. Hum Reprod 2002;17:1663-8.

12. Orji EO, Kuti 0, Fasubaa OB. Impact of infertility on marital life in Nigeria. Int J Gynaecol Obstet 2002; 79:61-2.

13. Sundby J, Mboge R, Sonko S. Infertility in the Gambia: frequency and health care seeking. Soc Sci Med 1998;46:891-9.

14. Umezulike AC, Efetie ER. The psychological trauma of infertility in Nigeria. Int J Gynaecol Obstet 2004;84:178-80.

15. Favot I, Ngalula J, Mgalla Z, et al. HIV infection and sexual behaviour among women with infertility in Tanzania: a hospital-based study. Int J Epidemiol 1997;26:414-19.

16. Ikechebelu JI, Ikegwuonu SC, Joe-lkechebelu NN. HIV infection and sexual behaviour among infertile women in southeastern Nigeria. J Obstet Gynaecol 2002;22:306-7.

17. Chersich MF, Rees HV. Vulnerability of women in southern Africa to infection with HIV: biological determinants and priority health sector interventions. AIDS 2008;22 (Suppl 4):S27-40.

18. Kilmarx PH. Global epidemiology of HIV. Curr Opin HIV AIDS 2009:4:240-6

19. Kayirangwa E, Hanson J, Munyakazi L, et al. Current trends in Rwanda's HIV/AIDS epidemic. Sex Transm Infect 2006;82(Suppl 1):i27-31.

20. Schrijvers D, Delaporte E, Peeters M, et al. Seroprevalence of retroviral infection in women with different fertility statuses in Gabon, western equatorial Africa. J Acquir Immune Defic Syndr 1991:4:468-70.

21. Okonofua FE, Ako-Nai KA, Dighitoghi MD. Lower genital tract infections in infertile Nigerian women compared with controls. Genitourin Med 1995;71:163-8.

22. Gerrits T. Social and cultural aspects of infertility in Mozambique. Patient Educ Couns 1997;31:39-48. 